Article

\title{
Correlation Analysis of Phenolic Contents and Antioxidation in Yellow- and Black-Seeded Brassica napus
}

\author{
Yue Wang, Guisheng Meng, Sailing Chen, Yajie Chen, Jinjin Jiang * and You-Ping Wang * \\ Jiangsu Provincial Key Laboratory of Crop Genetics and Physiology, Yangzhou University, Yangzhou 225009, \\ China; 18252746287@163.com (Y.W.); mengguishengmgs@126.com (G.M.); 18852721748@163.com (S.C.); \\ chenyajie008@163.com (Y.C.) \\ * Correspondence: jjjiang@yzu.edu.cn (J.J.); wangyp@yzu.edu.cn (Y.-P.W.); Tel.: +86-514-87997303 (J.J. \& Y.-P.W.); \\ Fax: +86-514-87991747 (J.J. \& Y.-P.W.)
}

Received: 8 July 2018; Accepted: 18 July 2018; Published: 21 July 2018

\begin{abstract}
Brassica napus L. is rich in phenolic components and it has natural antioxidant characteristics which are important to human health. In the present study, the total phenolic and flavonoid contents of developing seeds of yellow- and black-seeded B. napus were compared. Both phenolic and flavonoid contents were significantly higher at 5 weeks after flowering (WAF) in black seeds (6.44 $\pm 0.97 \mathrm{mg}$ $\mathrm{EE} / \mathrm{g}$ phenolics and $3.78 \pm 0.05 \mathrm{mg} \mathrm{EE} / \mathrm{g}$ flavonoids) than yellow seeds $(2.80 \pm 0.13 \mathrm{mg} / \mathrm{g}$ phenolics and $0.83 \pm 0.01 \mathrm{mg} / \mathrm{g}$ flavonoids). HPLC-DAD-ESI/MS analysis revealed different content of 56 phenolic components between yellow and black-seeded B. napus, including kaempferol-3-O-glucoside, isorhamnetin-3-O-glucoside, quercetin-3-O-sophoroside, procyanidin B2 ([DP 2]), which were significantly reduced in yellow seeds compared with black seeds. Applying the 2,2-diphenyl-1-picrylhydrazyl (DPPH) and 2,2'-azinobis-(3-ethylbenzothiazoline-6-sulfonic acid) (ABTS) radical assay, we found maximum clearance of DPPH and ABTS in the late developmental stages of yellow and black seeds. Additionally, the ferric reducing antioxidant power (FRAP) value maximized at 5 WAF in black seeds $(432.52 \pm 69.98 \mu \mathrm{mol} F(\mathrm{II}) / \mathrm{g}$ DW) and 6 WAF in yellow seeds (274.08 $\pm 2.40 \mu \mathrm{mol} F e(\mathrm{II}) / \mathrm{g}$ DW). Generally, antioxidant ability was significantly reduced in yellow-seeded B. napus compared to black rapeseed, and positive correlations between antioxidation and flavonoid content were found in both yellow- and black-seeded B. napus.
\end{abstract}

Keywords: Brassica napus; yellow seed; phenolic components; flavonoids; antioxidation

\section{Introduction}

Rapeseed (Brassica napus L.) is the most broadly cultivated Brassica species in the world for its high oil $(\sim 50 \%)$ and protein $(\sim 25 \%)$ content [1]. As one of the important oil crops for both edible and industrial oil, B. napus needs to improve its nutritional value and agronomic yield as the current output is insufficient to meet demand in China [2]. Glucosinolates and sinapate esters are two major anti-nutritional compounds in rapeseed, thus, over the past few decades breeders have put tremendous effort into selecting double-low cultivars (that is, varieties with low-glucosinolate and low-erucic acid) [3]. High concentration of glucosinolates in seeds of Brassica crops reduces the nutritional value of seed meal as protein-rich fodder, since their hydrolytic products (e.g., thiocyanate, oxazolidine-2-thiones) interact with the thyroid gland and cause metabolic disturbances [4]. Recently, mutation of genes encoding glucosinolate transporters has reduced the anti-nutritional glucosinolates in Brassica oilseeds [5]. Yellow-seeded B. napus has been evaluated as having significant advantages over black rapeseed, such as improved nutrients (oil and protein), and reduced anti-nutrients (phenolic compounds, lignin and fiber). These anti-nutrients are not 
beneficial for oil and seed meal production [6-10]. Hitherto, yellow-seeded B. napus were mainly bred by interspecific hybridization of Brassica [11], Li et al. first reported yellow rapeseeds from somatic hybridization of B. napus-Sinapis alba [12]. On the other hand, Brassicaceae crops are well known for their enriched secondary metabolites, especially for phytochemicals with antioxidant activity, including derivatives of hydroxycinnamic acids, sinapic acids, flavonols and anthocyanins [13]. Of these, the accumulation of anthocyanins is responsible for the red, blue, and purple colors in plant species [14]. It has been confirmed that these antioxidant compounds are helpful in preventing cardiovascular, heart disease and cancer by modulating some signaling pathways in mammalian cells [15-20]. The medical functions of polyphenols were mainly due to the antioxidant activity, although the mechanism of each polyphenol is not fully understood. In Aronia prunifolia hybrids, cyanidin glycosides have been proved to inhibit HeLa human cervical tumor cell proliferation [21]. However, these chemicals greatly reduce the quality of rapeseed oil and meal [22]. Rich phenolics in rapeseeds greatly hinder the use of rapeseed meal for feeding animals since most insoluble flavonoids, especially proanthocyanidins (PAs), can impair the digestibility of seed meal [23]. Phenolic compounds (e.g., sinapoyl esters and PAs) are responsible for the dark color and bitter taste of rapeseed meal and derived protein products, and they are one of the principle factors hampering the use of rapeseeds $[13,24]$. The breeding of rapeseed with reduced or increased phenolics depends on its main economic use, that is, seed oil/animal fodder or edible vegetable.

The characteristics of yellow-seeded rapeseed are correlated to the variation in phenolic compound synthesis and accumulation $[8,25]$. The pathways responsible for phenylpropanoid metabolism and flavonoid biosynthesis have been well elucidated in Arabidopsis and Brassica, providing multiple phenolic compounds (e.g., flavonoid and hydroxycinnamic acid derivatives) [26,27]. Phenolic compounds are difficult to fully identify due to their similar retention time and the limitations of detection technology (e.g., classic high-performance liquid chromatography, HPLC) [23,28]. Yang et al. reported a profile of hydrolysable tannins and other phenolic compounds in emblic leafflower fruits by HPLC-diode array detector (DAD)-electrospray ionization (ESI)-mass spectrometer (MS) analysis [29]. Recently, Shao et al. provided a comprehensive description of phenolic compounds in mature seeds of B. napus (black seed) [30]. This study provided the first detailed comparison of phenolic compounds in developing seeds of yellow and black rapeseed via HPLC-photodiode array detector (PDA)-ESI(-)/MS. The yellow rapeseed used in the present study is an introgression line selected from progenies of B. napus-S. alba somatic hybrids [12]. The black rapeseed is the backcrossing parent used for B. napus-S. alba hybrids. The antioxidant activity of developing rapeseeds were analyzed and correlated with phenolic content. The comprehensive accumulation pattern of phenolic compounds in developing rapeseeds, accompanied by analysis of the correlation between phenolic content and antioxidant activity, will help to elucidate the character of yellow rapeseeds, the variation in seed color related gene expression, and provide guidance for rapeseed breeding.

\section{Results and Discussion}

\subsection{Comparison of Total Phenolic and Flavonoid Content in Developing Seeds of Yellow-and Black-Seeded B. napus}

We found that both total phenolic and flavonoid content in black rapeseed maximized at 5 weeks after flowering (WAF) and declined thereafter, whereas, total phenolic and flavonoid content continued to increase as the yellow seeds developed (Figure 1). Also, total phenolic and flavonoid content were significantly higher throughout black seed development (except for mature seeds) than yellow seed. This agrees with the accumulation pattern reported by Jiang et al. [8]. Qu et al. reported that polymeric phenolic compounds started accumulating at 21 days after pollination (DAP), and a significant difference between yellow and black rapeseed was observed from 28 DAP to 49 DAP [25]. 

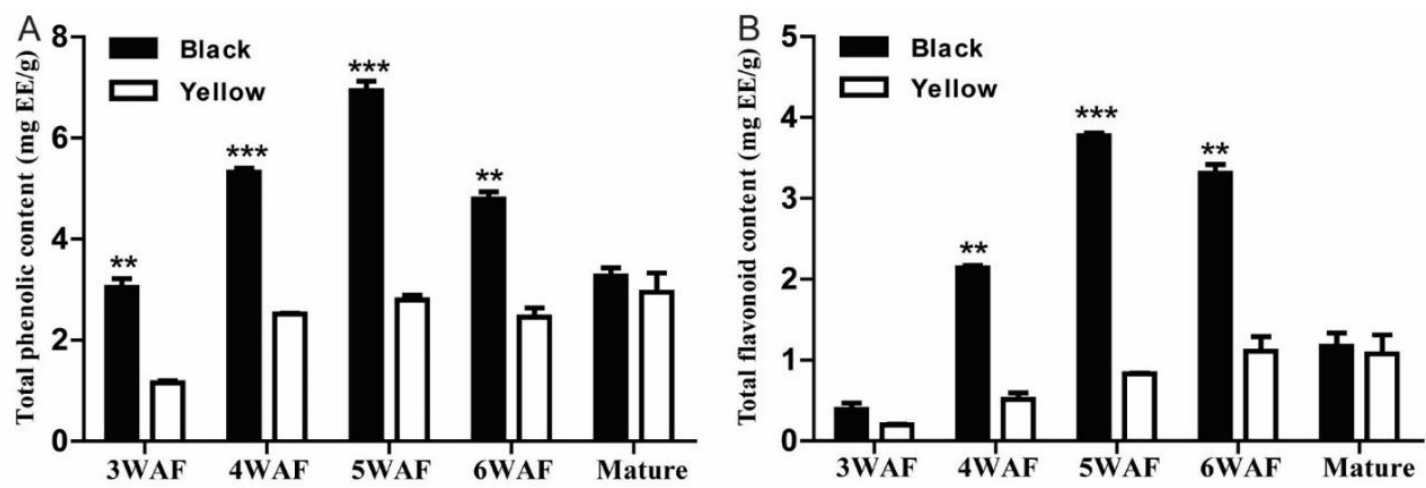

Figure 1. Total phenolic and flavonoid content in developing seeds of yellow- and black-seeded B. napus. (A). Total phenolic content; (B). Total flavonoid content. ${ }^{* *}$, and ${ }^{* * *}$ indicate significance at $p<0.05,0.01$ and 0.001, respectively. WAF, week after flowering; EE, epicatechin equivalents.

\subsection{HPLC-DAD-ESI/MS Analysis of Phenolic Compounds in Yellow- and Black-Seeded B. napus}

Previously, Shao et al. reported a comprehensive polyphenolic profile of B. napus [30]. Here, we applied the assay in a detailed comparison of hydroxycinnamic acid derivative and flavonoid content between yellow- and black-seeded B. napus. A total of 56 chemical compounds were detected, with several differences found during yellow and black seed development (Figure 2; Supplementary Table S1), including 15 hydroxycinnamic acid derivatives, 21 kaempferols (km), 10 isorhamnetins (is), 5 quercetins (qn), 5 epicatechin and derivatives. We found most of the hydroxycinnamic acid derivatives, including trans-sinapic acid, different isomers of sinapoylhexose, disinapoylglucoside, and trisinapoylgentiobiose, were more accumulated in yellow seed compared with black seed. The amount of cis-sinapic acid in the early developmental stages of yellow seed was nearly twice of the black seed content, and trans-sinapic acid was significantly higher in matured yellow seed than black seed. All the isomers of sinapoylhexose were much more accumulated in the yellow-seeded B. napus than the black-seeded B. napus. 1,2-disinapoylglucoside, 1,6-disinapoylglucoside, and two isomers of trisinapolygentiobiose accumulated with seed development, which were maximized in mature seeds and were significantly higher in matured yellow seed than black seed. One isomer of disinapoylgentiobiose was more accumulated in matured yellow seed than black seed, whereas another two isomers of disinapoylgentiobiose were less accumulated in yellow seed than black seed. Also, we found two putative hydroxycinnamic acid derivatives were more accumulated at late developmental stages of yellow seed than black seed (Figure 3).

As shown in Figure 4, we found most of the kaempferol derivatives were reduced throughout yellow seed development than black seed, except for km-3-Osophoroside-7-glucoside, km-3-O-sophoroside, $\mathrm{km}-3-\mathrm{O}$-triglucoside-7-O-glucoside, km-3-Osinapoylsophorotrioside-7-O-glucoside, $\mathrm{km}$-3-O-sinapoyldiglucoside-7-O-sinapoylglucoside, km-3-O-glucoside-7-O-glucoside, km-3-O-sinapoylsophoroside-7-O-sinapoylglucoside, km-3-Osinapoylsophoroside-7-O-sinapoylglucoside*, km-3-O-feroloylsophoroside-7-O-glucoside, km-3-Odisinapoylgalloyldiglucoside, which were higher at specific developmental stages of yellow seed compared to black seed. Besides, similar accumulation patterns of kaempferol derivatives were observed in both yellow and black rapeseeds. For instance, km-3-O-caffeoyldiglucoside-7-O-glucoside accumulated with seed development, $\mathrm{km}-3-\mathrm{O}$-sophoroside reduced with seed development, whereas $\mathrm{km}$-3-O-diglucoside maximized at 5 WAF. 

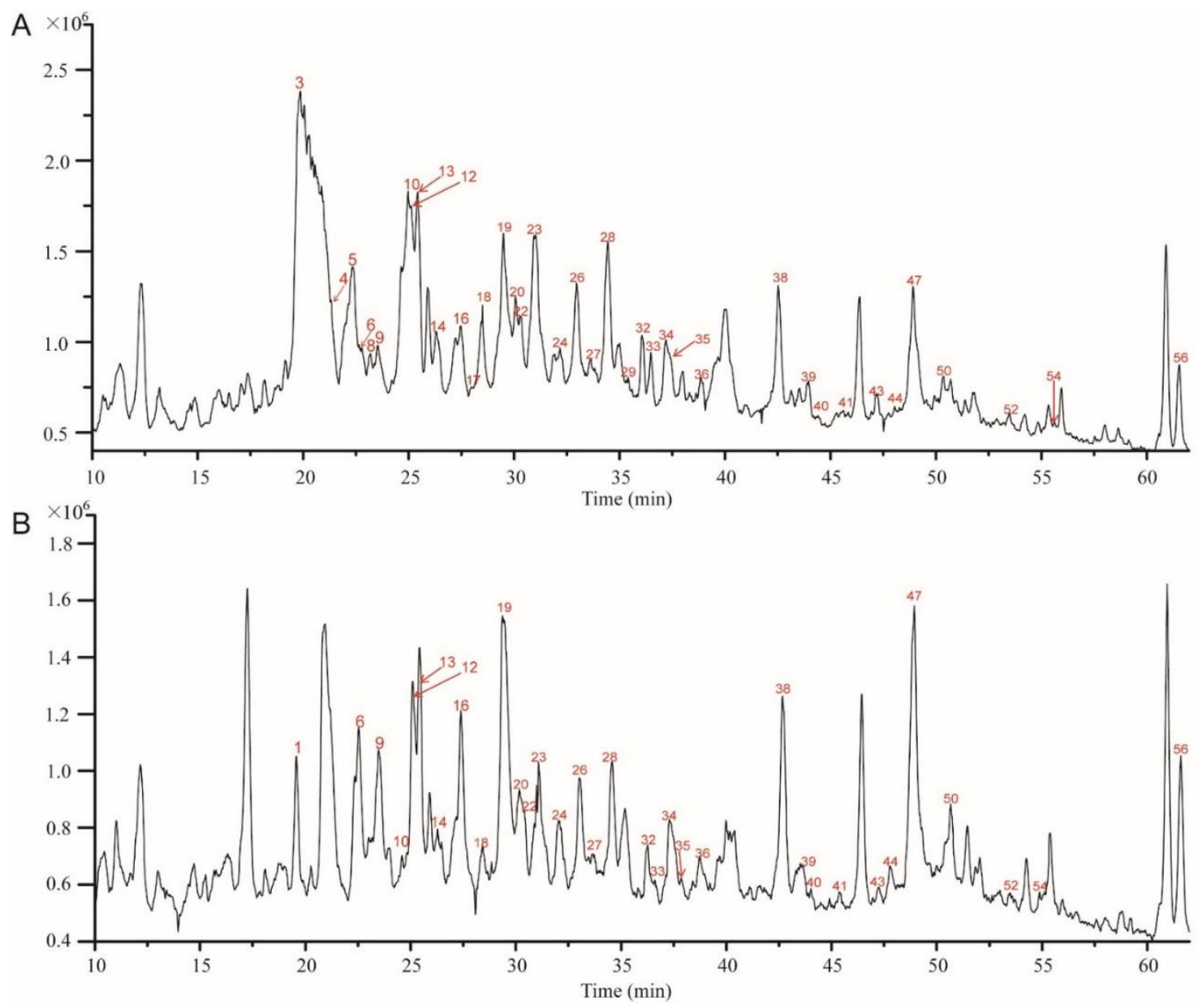

Figure 2. Liquid chromatography-electrospray ionization mass spectrometry (LC-ESI-MS) profile of phenolics from 5 WAF of yellow and black rapeseed. (A) black seed; (B) yellow seed. Peaks: (1) qn-3-O-diglucoside-7-O-glucoside; (3) km-3-O-sophoroside-7-O-glucoside; (4) sinapoylhexose; (5) procyanidin B2 ([DP 2]); (6) km-3-O-caffeoylsophoroside-7-O-glucoside; (8) qn-3-O-sinapoylsophoroside-7-O-glucoside; (9) sinapoylhexose*; (10) (-)-epicatechin; (12) km-3-O-sinapoylsophorotrioside-7-O-glucoside; (13) km-3-O-sinapoylsophoroside-7-O-glucoside; (14) km-3-O-feruloylsophoroside-7-O-glucoside; (16) is-3-O-glucoside-7-O-glucoside; (17) sinapoylhexose ${ }^{* * *}$; (18) [DP 3]; (19) km-3-O-sophoroside; (20) is-O-diglucoside-sulfate; (22) qn-3-O-sophoroside; (23) km-3-O-diglucoside; (24) cis-sinapic acid; (26) is-3-O-diglucoside; (27) km-3-O-disinapoylgalloyldiglucoside; (28) km-7-O-sophoroside; (29) qn-3-O-sinapoylsophoroside-7-O-glucoside*; (32) km-3-O-sinapoyldiglucoside-7-O-glucoside; (33) is-3-O-sinapoyldiglucoside-7-O-glucoside; (34) trans-sinapic acid; (35) km-3-O-sinapoylsophoroside; (36) [DP 3]*; (38) is-3-O-sinapoylglucoside-sulfate-7-O-glucoside; (39) km-3-O-glucoside; (40) is-3-O-glucoside; (41) km-3-O-sophoroside-7-O-sinapoylglucoside; (43) disinapoylgentiobiose*; (44) is-3-O-sinapoylglucoside-7-O-glucoside; (47) disinapoylgentiobiose ${ }^{* *}$; (50) putative hydroxycinnamic acid derivative; (52) 1,2,2'-trisinapoylgentiobiose; (54) 1,6-disinapoylglucoside; (56) putative quercetin. $\mathrm{km}$, kaempferol; is, isorhamnetin; qn, quercetin; DP, degree of polymerization of the epicatechin unit. * indicates for different isomers. 


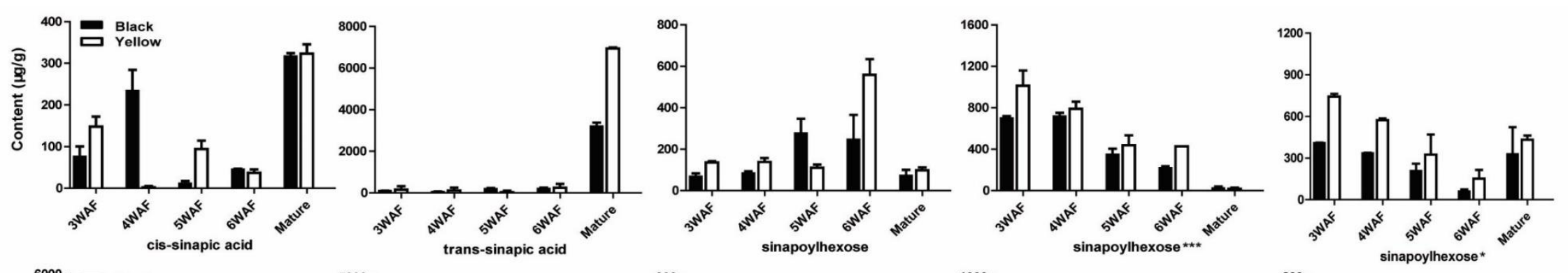
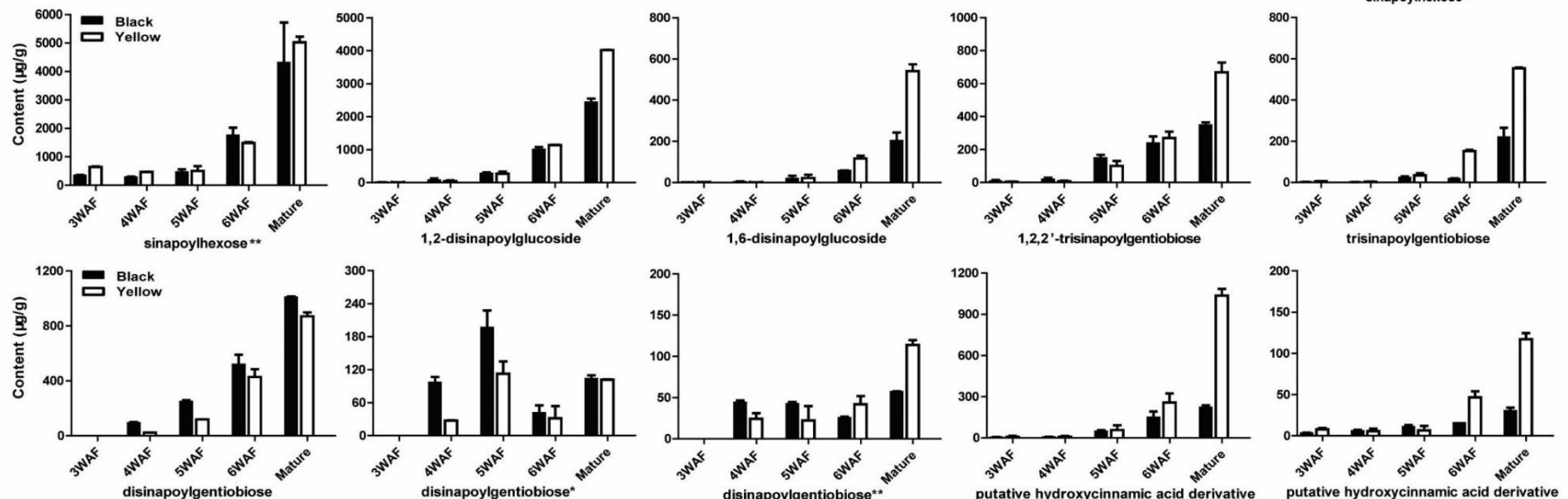

Figure 3. Accumulation pattern of hydroxycinnamic acid derivatives in yellow and black rapeseed. WAF, week after flowering. 

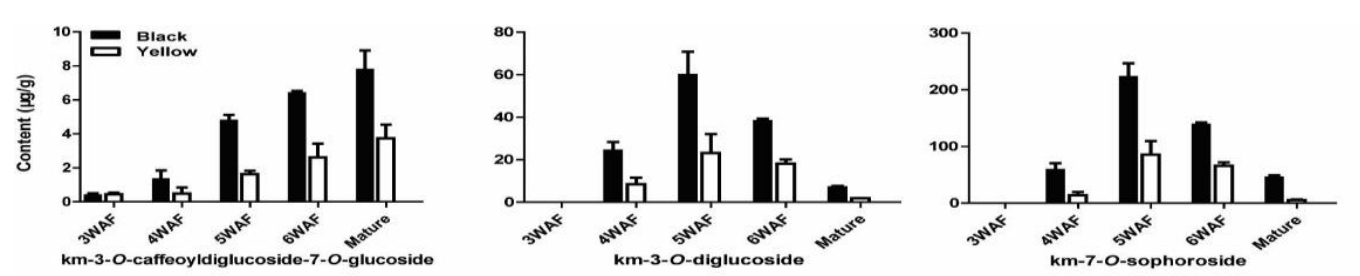

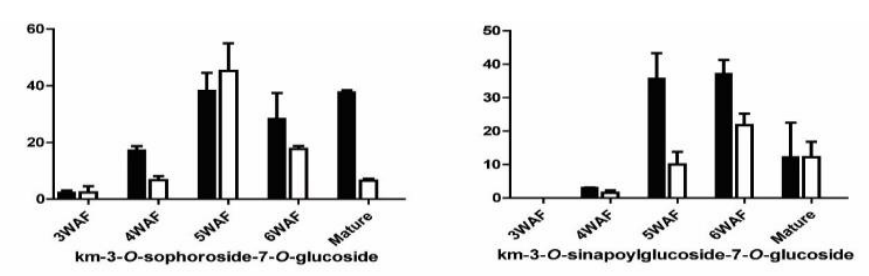
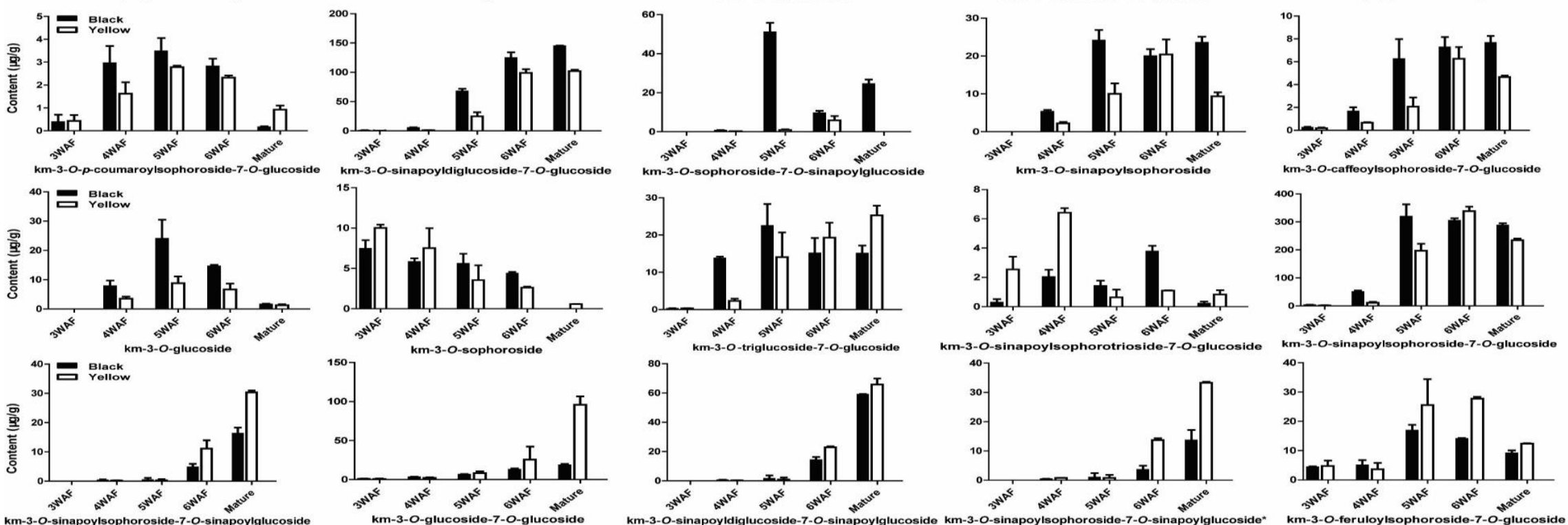

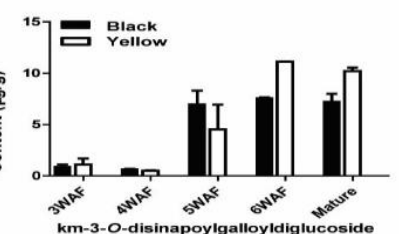

Figure 4. Accumulation pattern of kaempferols in yellow and black rapeseed. km, kaempferol; WAF, week after flowering. 
As illustrated in Figure 5, ten isorhamnetins were shown to have different content in the two rapeseeds, including is-3-O-glucoside, is-3-O-glucoside-sulfate, is-3-O-glucoside-7-O-glucoside, is-3-O-glucoside-7-O-acetylglucoside, is-3-O-diglucoside, is- $O$-diglucoside-sulfate, is-3-Osinapoyldiglucoside-7-O-glucoside, is-3-O-sinapoylglucoside-7-O-glucoside which were less accumulated in yellow seed than black seed. However, is-3-O-sinapoylglucoside-7-O-glucoside* and is-3-O-sinapoylglucoside-sulfate-7-O-glucoside were more accumulated at later developmental stages of yellow seed than black seed.

Four quercetins were also reduced in yellow seed compared with black seed, including qn-3-O-sophoroside, qn-3-O-diglucoside-7-O-glucoside, qn-3-O-sinapoylsophoroside-7-O-glucoside, and qn-3-O-sinapoylsophoroside-7-O-glucoside*. At $3 \mathrm{WAF}$, qn-3-O-diglucoside-7-O-glucoside in yellow rapeseed was higher than black rapeseed (Figure 6). Besides, epicatechin and its four derivatives were significantly reduced throughout yellow seed development, including procyanidin B2 ([DP 2]), [DP 3], [DP 3]*, [DP 4] (Figure 6).

Previously, Auger et al. identified 13 flavonoids in developing seeds of eight black-seeded B. napus genotypes [23]. Jiang et al. reported 19 compounds when comparing polyphenolics in different yellow seed lines and black rapeseed [8]. Qu et al. detected 35 main flavonols in rapeseed and found 2 and 13 unique flavonol derivatives in black and yellow rapeseed, respectively [25]. This is the first comprehensive comparison of flavonoids and hydroxycinnamic acid derivatives between yellow and black rapeseed, which will be helpful in explaining the characteristics of yellow-seeded B. napus. We were also able to identify a more detailed accumulation pattern of polyphenolics in the developing seeds of $B$. napus. We found that hydroxycinnamic acid derivatives were significantly higher than flavonoid content, even though flavonoids were more accumulated at 4-6 WAF compared to 3 WAF and the mature stage (Figure 7A). Among the four groups of flavonoids, the amount of kaempferol and epicatechin was higher than isorhamnetin and quercetin (Figure 7B). Epicatechin was mostly accumulated at 4-6 WAF in black rapeseed, but not in yellow rapeseed. The total content of kaempferol in yellow seed was less than black seed, although some of the derivatives with low content were higher at specific stages of yellow seed development compared to black seed. As to quercetin, it was more accumulated in yellow seed after $4 \mathrm{WAF}$, mainly due to an unknown compound detected with a parent ion at $m / z$ 301, which has not been reported before. Also, the content of this putative quercetin was much higher than other quercetins, e.g., $106 \mu \mathrm{g} / \mathrm{g}$ and $212 \mu \mathrm{g} / \mathrm{g}$ at 5 WAF for black and yellow seeds, respectively. 

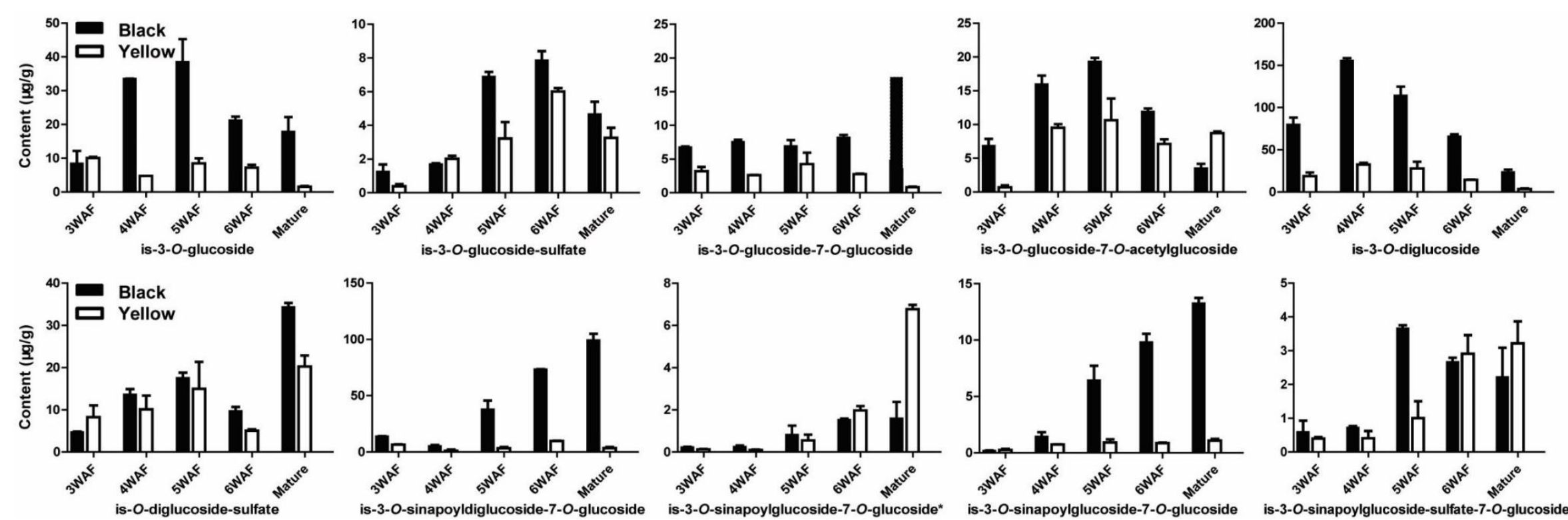

Figure 5. Accumulation pattern of isorhamnetins in yellow and black rapeseed. is, isorhamnetin; WAF, week after flowering. 


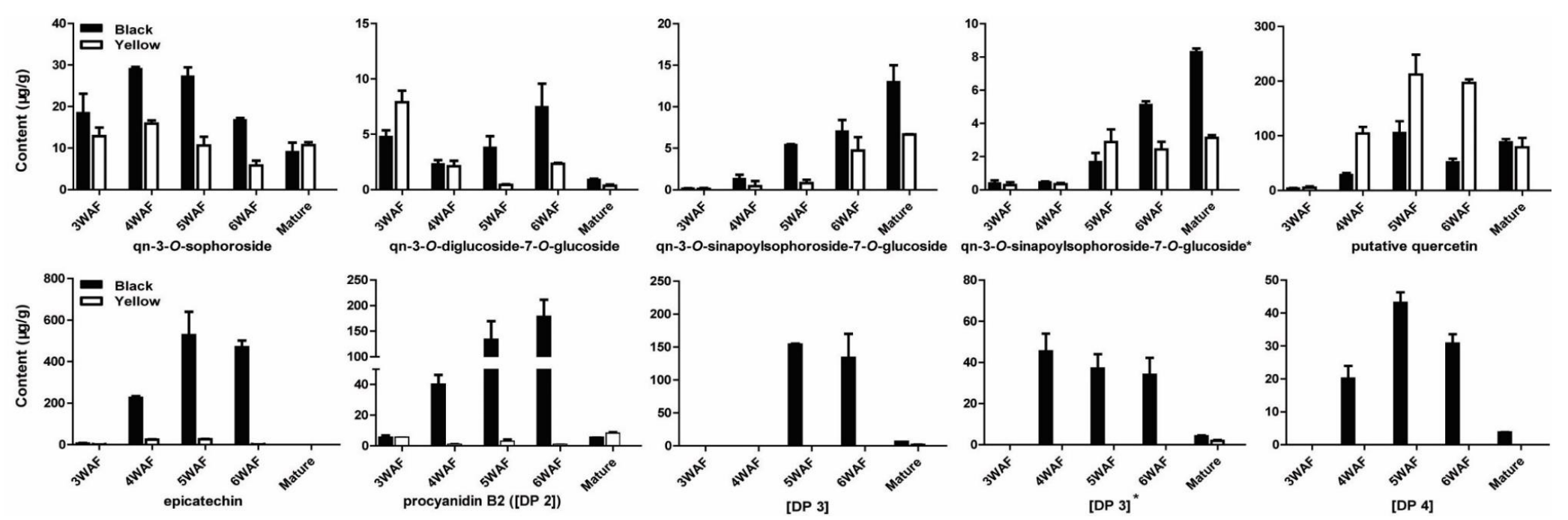

Figure 6. Accumulation pattern of quercetins, epicatechin and derivatives in yellow and black rapeseed. qn, quercetin; DP, degree of polymerization of the epicatechin unit; WAF, week after flowering.
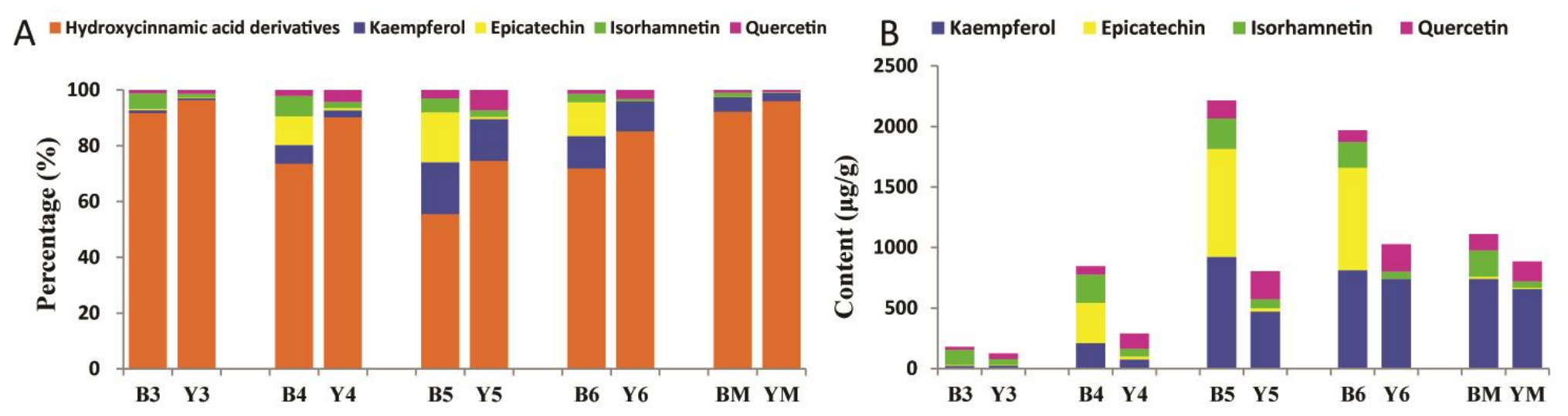

Figure 7. Time-course accumulation pattern of main phenolic components in developing seeds of yellow- and black-seeded B. napus. (A) Percentage of phenolic components; (B) Content of flavonoids. 


\subsection{Antioxidant Activity of Yellow- and Black-Seeded B. napus}

Different methods were used to assess the antioxidant activity of phenolic compounds (Table 1). The 2,2-diphenyl-1-picrylhydrazyl (DPPH) scavenging activity in yellow rapeseed was significantly lower than black rapeseed throughout seed development except for the mature stage. Similar differences were identified in 2,2'-azinobis-(3-ethylbenzothiazoline-6-sulfonic acid) (ABTS) radical assay. The DPPH radical scavenging ability (RSA) in 4 WAF black seed was 14 times that of yellow seed, and the ABTS radical cation scavenging ability (RCSA) at the same developmental stage was 2.5 times greater in black seed compared with yellow seed. Additionally, different patterns for DPPH and ABTS were observed between yellow and black seeds, which agreed with the phenolic accumulation in the different rapeseeds. As to the ferric reducing antioxidant power (FRAP) assay, we found the polyphenolic extracts from developing black rapeseed exhibited significantly higher reducing power at 3-6 WAF compared with yellow rapeseed. The most significant difference was observed at 4 WAF, $375.38 \mu \mathrm{M}$ Fe (II)/g DW in black seed and 112.29 $\mu \mathrm{M}$ Fe (II)/g DW in yellow seed. All this data revealed higher antioxidant activity in black rapeseed than yellow rapeseed. Previously, the antioxidant activity and the abundant polyphenolics in Brassicas, especially vegetable plants, have been recognized as having putative medical functions in preventing diseases [31,32]. Antioxidant capacity has also been identified in seed meals of B. juncea and canola for better extraction of natural antioxidants [33]. This is the first comparison of the antioxidant activity of phenolics from developing seeds of yellow and black rapeseed.

Table 1. Antioxidant activities of black and yellow seeds determined by DPPH, ABTS and FRAP assays.

\begin{tabular}{ccccccc}
\hline & \multicolumn{2}{c}{ DPPH (\%) } & \multicolumn{2}{c}{ ABTS (\%) } & \multicolumn{2}{c}{ FRAP $(\mu \mathrm{moL}$ Fe (II)/g DW) } \\
\cline { 2 - 6 } & Black Seed & Yellow Seed & Black Seed & Yellow Seed & Black Seed & Yellow Seed \\
\hline 3 WAF & $18.12749^{\mathrm{a}}$ & $1.593625^{\mathrm{b}}$ & $38.36^{\mathrm{a}}$ & $15.36^{\mathrm{b}}$ & $246.48^{\mathrm{a}}$ & $105.32^{\mathrm{b}}$ \\
4 WAF & $52.29084^{\mathrm{a}}$ & $3.685259^{\mathrm{b}}$ & $75.79^{\mathrm{a}}$ & $21.79^{\mathrm{b}}$ & $375.38^{\mathrm{a}}$ & $112.29^{\mathrm{b}}$ \\
5 WAF & $78.18725^{\mathrm{a}}$ & $28.68526^{\mathrm{b}}$ & $98.36^{\mathrm{a}}$ & $54.07^{\mathrm{b}}$ & $432.52^{\mathrm{a}}$ & $236.95^{\mathrm{b}}$ \\
6 WAF & $81.57371^{\mathrm{a}}$ & $41.43426^{\mathrm{b}}$ & $98.36^{\mathrm{a}}$ & $75.50^{\mathrm{b}}$ & $357.46^{\mathrm{a}}$ & $274.08^{\mathrm{b}}$ \\
Mature & $55.07968^{\mathrm{b}}$ & $68.02789^{\mathrm{a}}$ & $95.50^{\mathrm{a}}$ & $98.07^{\mathrm{a}}$ & $330.52^{\mathrm{a}}$ & $313.55^{\mathrm{b}}$ \\
\hline
\end{tabular}

Data represent the mean \pm standard error $(n=3) .{ }^{\mathrm{a}}$ and ${ }^{\mathrm{b}}$ indicate a significant difference at $p<0.05$ level. WAF, week after flowering.

\subsection{Correlation Analysis of Phenolic Compounds and Antioxidant Activity}

Based on the assay of phenolic contents and antioxidant activity of yellow and black rapeseed, correlation coefficients between antioxidant activity and total phenolic/flavonoid content were analyzed (Table 2). We found that the content of phenolics and flavonoids were closely correlated in yellow (0.897) and black rapeseed (0.805), since flavonoid is a major group of phenolics [13]. This is in line with previous reports on lupin and mung beans $[16,34]$. The three indexes of antioxidant activity were more highly correlated with flavonoid content than phenolic content in the two rapeseed lines, indicating that flavonoids are more related to the antioxidant activity in rapeseed. This agrees with previous reports that Brassica vegetables (e.g., broccoli, red cabbage, and brussels sprouts) have the highest antioxidant capability due to high levels of anthocyanins [35]. Farag et al. classified the phenolic metabolites in different organs of B. napus and found the antioxidant activity was correlated to some extent with total flavonoid content [28]. Teh et al. reported canola seed cake with higher polyphenols than hemp and flax seed cakes, which was positively correlated with antioxidative capacity [36]. He et al. found the total antioxidant activity in purple Chinese cabbage was highly positively correlated with the anthocyanin content [37]. Thus, the correlation between flavonoids and antioxidant activity in rapeseed is reasonable and could help to explain the different quality of yellow and black rapeseeds. 
Table 2. Correlation coefficient analysis of phenolic compounds and antioxidant activity in developing seeds of yellow- and black-seeded B. napus.

\begin{tabular}{ccccccccc}
\hline & \multicolumn{2}{c}{ Flavonoids } & \multicolumn{2}{c}{ ABTS } & \multicolumn{2}{c}{ DPPH } & \multicolumn{2}{c}{ FRAP } \\
\hline & Black & Yellow & Black & Yellow & Black & Yellow & Black & Yellow \\
& Seed & Seed & Seed & Seed & Seed & Seed & Seed & Seed \\
\hline Phenolics & $0.897^{*}$ & $0.8053^{*}$ & 0.535 & 0.695 & 0.713 & 0.607 & $0.921^{*}$ & 0.701 \\
Flavonoids & - & - & 0.747 & $0.936^{*}$ & $0.923^{*}$ & $0.889 *$ & $0.889^{*}$ & $0.948^{*}$ \\
\hline
\end{tabular}

\section{Experimental Section}

\subsection{Plant Materials}

Yellow-seeded B. napus selected from progenies of somatic hybrids between B. napus and S. alba, and the black-seeded B. napus were cultivated in the experimental field of Yangzhou university. Seeds from each five siliques of five plants were harvested at $3 \mathrm{WAF}, 4 \mathrm{WAF}, 5 \mathrm{WAF}, 6 \mathrm{WAF}$ and mature stage. All the samples were frozen at $-80^{\circ} \mathrm{C}$ for use.

\subsection{Extraction of Seed Polyphenols}

According to Shao et al. [30], 100 seeds from each developmental stage were dried and weighed for preparing crude extracts of polyphenols. After grinding with $5 \mathrm{~mL}$ methanol/acetone/water $(40: 40: 20, v / v / v)$ for $5 \mathrm{~min}$, samples were sonicated for $15 \mathrm{~min}$ and centrifuged at 12,000 rpm for $10 \mathrm{~min}$. The supernatants were collected and combined with supernatants from re-extractions of the pellets. All the crude extracts were concentrated to $1 \mathrm{~mL}$ and stored at $-80^{\circ} \mathrm{C}$ for chemical measurements.

\subsection{Quantification of Soluble Phenolic Acids and Flavonoids}

Total content of soluble phenolics was measured according to Jiang et al., using the Folin-Ciocalteu method [8]. Epicatechin was used for standard calibration. The total content of soluble flavonoids was measured according to Faudale et al. [38]. The crude polyphenol extract $(0.2 \mathrm{~mL})$ was diluted

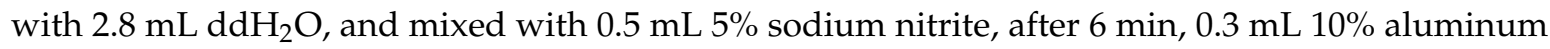
chloride was added for 5 min of reaction. Finally, the reaction solution was mixed with $1 \mathrm{~mL} 1 \mathrm{M}$ $\mathrm{NaOH}$ and $0.55 \mathrm{~mL} \mathrm{ddH_{2 }} \mathrm{O}$. The absorbance value was recorded at $510 \mathrm{~nm}$ using epicatechin as the standard calibration.

\subsection{HPLC-DAD-ESI/MS Analysis of Polyphenolic Components}

The polyphenol extracts were filtered through $0.45 \mu \mathrm{m}$ Teflon membrane before HPLC-ESI/MS ${ }^{2}$ analysis, using an Agilent 6460 system (Agilent, Santa Clara, CA, USA) coupled with automatic injector ALS, Quat Pump, 1260 thermostatted column compartment, 1260 DAD detector, and MS QQQ [8]. Generally, $10 \mu \mathrm{L}$ extracts were separated in a Ultimate XB-C18 column $(2.1 \times 150 \mathrm{~mm}$, $3.5 \mu \mathrm{m}$; Welch, Shanghai, China) accompanied with a C18 precolumn $(2 \times 4 \mathrm{~mm}, 3.5 \mu \mathrm{m}$; Phenomenex, Torrance, CA, USA). All the settings and elution steps were done as before. After a full scan of all the polyphenols, $\mathrm{MS}^{2}$ analysis was performed to confirm chemical structures under a $\mathrm{N}_{2}$ gas flow and voltage of 15-40 V. Both positive and negative ion mode and an $m / z$ range of 100-2000 were selected during ESI source operation. All the analyses was controlled with Agilent MassHunter Workstation Data Acquisition and data analysis was performed using Agilent MassHunter Qualitative Analysis. Confirmation of all the chemicals was based on the UV absorption characteristics, $\mathrm{MS}^{2}$ data and rapeseed polyphenol profile reported by Shao et al. [30]. Relative quantification of all the chemicals was based on the ion chromatograms under $[\mathrm{M}-\mathrm{H}]^{-}$or $[\mathrm{M}+\mathrm{H}]^{+}$, using standards (procyanidin B2, (-)-epicatechin, quercetin 3-O- $\beta$-D-glucoside, and isorhamnetin-3-O-glucoside) with similar aglycon cores for calibration. 


\subsection{Antioxidant Activity Analysis}

The antioxidant activity of polyphenol extracts was measured using DPPH, ABTS and FRAP methods with few modifications [39-41]. Polyphenol extracts $(100 \mu \mathrm{L})$ were mixed with $3.9 \mathrm{~mL}$ $100 \mu \mathrm{M}$ DPPH reaction solution, and the mixture was allowed $30 \mathrm{~min}$ of reaction in darkness and at room temperature. The absorbance was measured at $515 \mathrm{~nm}$ against a blank of DPPH solution. The DPPH RSA was calculated as DPPH RSA $(\%)=\left(1-\mathrm{A}_{515 \mathrm{~nm} \text { of sample }} / \mathrm{A}_{515 \mathrm{~nm} \text { of control }}\right) \times 100$. The reaction of DPPH and $50 \%$ methanol was used as control. For ABTS RCSA assay, $0.5 \mathrm{~mL}$ polyphenol extract was mixed with $2.5 \mathrm{~mL}$ ABTS solution containing $7.4 \mathrm{mM}$ ABTS and $2.6 \mathrm{mM}$ potassium persulfate, and allowed to react for $7 \mathrm{~min}$ in darkness and at $30^{\circ} \mathrm{C}$. The absorbance was measured at $734 \mathrm{~nm}$, using ABTS solution without samples as control. The antioxidant ability was calculated as ABTS RCSA $(\%)=\left[\left(\mathrm{A}_{734 \mathrm{~nm} \text { of control }}-\mathrm{A}_{734 \mathrm{~nm} \text { of sample }}\right) / \mathrm{A}_{734 \mathrm{~nm} \text { of control }}\right] \times 100$. For FRAP assay, $100 \mu \mathrm{L}$ polyphenol extracts were mixed with $2.4 \mathrm{~mL}$ FRAP reagent containing $0.3 \mathrm{M}$ acetate buffer (pH 3.6), $10 \mathrm{mM}$ 2,4,6-tri(2-pyridyl)-s-triazine (TPTZ) solution and $20 \mathrm{mM} \mathrm{FeCl}_{3}$ solution (10:1:1, v/v/v). After incubation at $37^{\circ} \mathrm{C}$ for $10 \mathrm{~min}$, the absorbance of $\mathrm{Fe}^{2+}-\mathrm{TPTZ}$ was measured at $593 \mathrm{~nm}$ against a blank containing $\mathrm{ddH}_{2} \mathrm{O}$ and FRAP reagent. The results were expressed as $\mu \mathrm{M} F$ (II)/g DW, in calibration against a standard curve of $\mathrm{FeSO}_{4}$ in different concentrations $(0.1,0.2,0.4,0.6,0.8$ and $1.0 \mathrm{mM})$.

\subsection{Statistics Analysis}

All measurements were carried out in triplicate in order to determine reproducibility, and the results are reported as mean \pm standard deviation. Correlation analysis of the results were performed with SPSS v.16.0. Statistical significance was defined at level of $p<0.05$.

\section{Conclusions}

Yellow rapeseed has been a major focus in B. napus breeding due to its quality advantages. Molecular analysis of Arabidopsis and Brassica has proved that this phenotype is correlated to the flavonoid biosynthetic pathway. Phenolic compounds, such as flavonols, anthocyanins, proanthocyanidins, are the main pigments associated with antioxidant activities. Also, this pathway shares common chemical precursors for phenylpropanoid biosynthesis, phenylalanine metabolism, which are related to lignin biosynthesis. Hitherto, comprehensive comparison of phenolic compounds in developing seeds of yellow- and black-seeded B. napus has not been reported. Based on HPLC-DAD-ESI/MS analysis, we carried out a comparison of phenolic compounds in yellow and black rapeseeds, involving all of the seed developmental stages. A detailed accumulation pattern of polyphenolics in B. napus is also provided. In addition, the antioxidant capability of developing seeds from two rapeseed lines was also detected and correlated with the content of phenolics and flavonoids. Obviously, flavonoid content is significantly correlated with the antioxidant ability of rapeseeds. This more comprehensive profile of phenolic accumulation in yellow and black rapeseeds, should be helpful in breeding rapeseeds with expected antioxidant quality since controversies may exist in breeding rapeseed with higher or lower content of flavonoids, depending on its economic value as seed oil/animal fodder or edible vegetable. With the support of gene expressional data for two rapeseed lines, and the reported expressional profile of flavonoid biosynthesis related genes in Brassicaceae [42], manipulation of genes related to the biosynthesis of specific phenolic compounds could be very helpful in rapeseed breeding.

Supplementary Materials: The following are available online, Table S1: List and details of flavonoids and hydroxycinnamic acid derivatives identified in yellow- and black-seeded B. napus.

Author Contributions: Y.W. and G.M. performed the experiments, S.C. and Y.C. sampled the materials, J.J. and Y.-P.W. designed the experiments and revised the manuscript. All the authors approved the final manuscript.

Funding: This study was supported by the National Natural Science Foundations $(31330057,31771825,31401414)$, the National Key Research and Development Program of China (2016YFD0101000, 2016YFD0102000), China 
Postdoctoral Science Foundation (2015T80591, 2014M561719), Jiangsu Postdoctoral Science Foundation (1401078B), the Natural Science Foundation of Jiangsu Province (BK20140478), the Postgraduate Research \& Practice Innovation Program of Jiangsu Province (KYLX16_1386, XKYCX17_066), the Undergraduate Training Program for Innovation and Entrepreneurship (x20170813), the Priority Academic Program Development of Jiangsu Higher Education Institutions, and Yangzhou University for Excellent Talent Support Program.

Acknowledgments: We sincerely thank the Test Center of Yangzhou University for the HPLC facilities.

Conflicts of Interest: The authors declare no conflict of interest.

\section{References}

1. Wolfram, K.; Schmidt, J.; Wray, V.; Milkowski, C.; Schliemann, W.; Strack, D. Profiling of phenylpropanoids in transgenic low-sinapine oilseed rapeseed (Brassica napus). Phytochemistry 2010, 71, 1076-1084. [CrossRef] [PubMed]

2. Liu, Q.; Wu, L.; Pu, H.; Li, C.; Hu, Q. Profiles and distribution of soluble and insoluble phenolics in Chinese rapeseed (Brassica napus). Food Chem. 2012, 135, 616-622. [CrossRef] [PubMed]

3. Fang, J.; Reichelt, M.; Kai, M.; Schneider, B. Metabolic profiling of lignins and other secondary metabolites from rapeseed (Brassica napus L.). J. Agric. Food Chem. 2012, 60, 10523-10529. [CrossRef] [PubMed]

4. Tayo, T.; Dutta, N.; Sharma, K. Effect of feeding canola quality rapeseed mustard meal on animal production-A review. Agric. Rev. 2012, 33, 114-121.

5. Nour-Eldin, H.H.; Madsen, S.R.; Engelen, S.; Jorgensen, M.E.; Olsen, C.E.; Andersen, J.S.; Seynnaeve, D.; Verhoye, T.; Fulawka, R.; Denolf, P.; et al. Reduction of antinutritional glucosinolates in Brassica oilseeds by mutation of genes encoding transporters. Nat. Biotechnol. 2016, 35, 377-385. [CrossRef] [PubMed]

6. Yu, C.Y. Molecular mechanism of manipulating seed coat coloration in oilseed Brassica species. J. Appl. Genet. 2013, 54, 135-145. [CrossRef] [PubMed]

7. Lipsa, F.D.; Snowdon, R.; Friedt, W. Quantitative genetic analysis of condensed tannins in oilseed rape meal. Euphytica 2012, 184, 195-205. [CrossRef]

8. Jiang, J.J.; Shao, Y.L.; Li, A.M.; Lu, C.L.; Zhang, Y.T.; Wang, Y.P. Flavonoid composition analysis and gene expression in developing seeds of yellow- and black-seeded Brassica napus. J. Integr. Plant Biol. 2013, 55, 537-551. [CrossRef] [PubMed]

9. Jiang, J.J.; Wang, Y.; Xie, T.; Rong, H.; Li, A.M.; Fang, Y.J.; Wang, Y.P. Metabolic characteristics in meal of black rapeseed and yellow-seeded progeny of Brassica napus-Sinapis alba hybrids. Molecules 2015, 20, 21204-21213. [CrossRef] [PubMed]

10. Liu, L.; Stein, A.; Wittkop, B.; Sarvari, P.; Li, J.; Yan, X.; Dreyer, F.; Frauen, M.; Friedt, W.; Snowdon, R.J. A knockout mutation in the lignin biosynthesis gene CCR1 ezplains a major QTL for acid detergent lignin content in Brassica napus seeds. Theor. Appl. Genet. 2012, 124, 1573-1586. [CrossRef] [PubMed]

11. Wen, J.; Zhu, L.X.; Qi, L.P.; Ke, H.M.; Yi, B.; Shen, J.X.; Tu, J.X.; Ma, C.Z.; Fu, T.D. Characterization of interploid hybrids from crosses between Brassica juncea and B. oleracea and the production of yellow-seeded B. napus. Theor. Appl. Genet. 2012, 125, 19-32. [CrossRef] [PubMed]

12. Li, A.; Jiang, J.; Zhang, Y.; Snowdon, R.J.; Liang, G.; Wang, Y. Molecular and cytological characterization of introgression lines in yellow seed derived from somatic hybrids between Brassica napus and Sinapis alba. Mol. Breed. 2012, 29, 209-219. [CrossRef]

13. Cartea, M.E.; Francisco, M.; Soengas, P.; Velasco, P. Phenolic compounds in Brassica vegetables. Molecules 2010, 16, 251-280. [CrossRef] [PubMed]

14. Zhang, Y.; Hu, Z.; Zhu, M.; Zhu, Z.; Wang, Z.; Tian, S.; Chen, G. Anthocyanin accumulation and molecular analysis of correlated genes in purple kohlrabi (Brassica oleracea var. gongylodes L.). J. Agric. Food Chem. 2015, 63, 4160-4169. [CrossRef] [PubMed]

15. Williams, R.J.; Spencer, J.P.; Rice-Evans, C. Flavonoids: Antioxidants or signaling molecules? Free Radic. Biol. Med. 2004, 36, 838-849. [CrossRef] [PubMed]

16. Pajak, P.; Socha, R.; Galkowska, D.; Roznowski, J.; Fortuna, T. Phenolic profile and antioxidant activity in selected seeds and sprouts. Food Chem. 2014, 143, 300-306. [CrossRef] [PubMed]

17. Rahnasto-Rilla, M.; Tyni, J.; Huovinen, M.; Jarho, E.; Kulikowicz, T.; Ravichandran, S.; Bohr, V.A.; Ferrucci, L.; Lahtela-Kakkonen, M.; Moaddel, R. Natural polyphenols as sirtuin 6 modulators. Sci. Rep. 2018, 8, 4163-4173. [CrossRef] [PubMed] 
18. Agudo, A.; Cabrera, L.; Amiano, P.; Ardanaz, E.; Barricarte, A.; Berenguer, T.; Chirlaque, M.D.; Dorronsoro, M.; Jakszyn, P.; Larranaga, N.; et al. Fruit and vegetable intakes, dietary antioxidant nutrients, and total mortality in spanish adults: Findings from the spanish cohort of the european prospective investigation into cancer and nutrition (EPIC-Spain). Am. J. Clin. Nutr. 2007, 85, 1634-1642. [CrossRef] [PubMed]

19. Higdon, J.V.; Delage, B.; Williams, D.E.; Dashwood, R.H. Cruciferous vegetables and human cancer risk: Epidemiologic evidence and mechanistic basis. Pharm. Res. 2007, 55, 224-236. [CrossRef] [PubMed]

20. Lam, T.K.; Gallicchio, L.; Lindsley, K.; Shiels, M.; Hammond, E.; Tao, X.G.; Chen, L.; Robinson, K.A.; Caulfield, L.E.; Herman, J.G.; et al. Cruciferous vegetable consumption and lung cancer risk: A systematic review. Cancer Epidemiol. Biomark. Prev. 2009, 18, 184-195. [CrossRef] [PubMed]

21. Rugina, D.; Sconta, Z.; Leopold, L.; Pintea, A.; Bunea, A.; Socaciu, C. Antioxidant activities of chokeberry extractes and the cytotoxic action of their anthocyanin fraction in HeLa human cervical tumor cells. J. Med. Food 2012, 15, 700-706. [CrossRef] [PubMed]

22. Liu, J.; Hua, W.; Yang, H.; Guo, T.; Sun, X.; Wang, X.; Liu, G.; Wang, H. Effects of specific organs on seed oil accumulation in Brassica napus L. Plant Sci. 2014, 227, 60-68. [CrossRef] [PubMed]

23. Augar, B.; Marnet, N.; Gautier, V.; Maia-Grondard, A.; Leprince, F.; Renard, M.; Guyot, S.; Nesi, N.; Routaboul, J.M. A detailed survey of seed coat flavonoids in developing seeds of Brassica napus L. J. Agric. Food Chem. 2010, 58, 6246-6256. [CrossRef] [PubMed]

24. Badani, A.G.; Snowdon, R.J.; Wittkop, B.; Lipsa, F.D.; Baetzel, R.; Horn, R.; De Haro, A.; Font, R.; Luhs, W.; Friedt, W. Colocalization of a partially dominant gene for yellow seed colour with a major QTL influencing acid detergent fibre (ADF) content in different crosses of oilseed rape (Brassica napus). Genome 2006, 49, 1499-1509. [CrossRef] [PubMed]

25. Qu, C.; Fu, F.; Lu, K.; Zhang, K.; Wang, R.; Xu, X.; Wang, M.; Lu, J.; Wan, H.; Tang, Z.; et al. Differential accumulation of phenolic compounds and expression of related genes in black- and yellow-seeded Brassica napus. J. Exp. Bot. 2013, 64, 2885-2898. [CrossRef] [PubMed]

26. Fraser, C.M.; Chapple, C. The phenylproponoid pathway in Arabidopsis. Arabidopsis Book 2011, 9 , e0152. [CrossRef] [PubMed]

27. Mittasch, J.; Bottcher, C.; Frolov, A.; Strack, D.; Milkowski, C. Reprogramming the phenylpropanoid metabolism in seeds of oilseed rape by suppressing the orthologs of REDUCED EPIDERMAL FLUORESCENCE1. Plant Physiol. 2013, 161, 1656-1669. [CrossRef] [PubMed]

28. Farag, M.A.; Eldin, M.G.S.; Kassem, H.; Fetouh, M.A.E. Metabolome classification of Brassica napus organs via UPLC-QTOF-PDA-MS and their anti-oxidant potential. Phytochem. Anal. 2013, 24, 277-287. [CrossRef] [PubMed]

29. Yang, B.; Kortesniemi, M.; Liu, P.Z.; Karonen, M.; Salminen, J.P. Analysis of hydrolysable tannins and other phenolic compounds in emblic leafflower (Phyllanthus emblica L.) fruits by high performance liquid chromatography-electrospray ionization mass spectrometry. J. Agric. Food Chem. 2012, 60, 8672-8683. [CrossRef] [PubMed]

30. Shao, Y.; Jiang, J.; Ran, L.; Lu, C.; Wei, C.; Wang, Y. Analysis of flavonoids and hydroxycinnamic acid derivatives in rapeseeds (Brassica napus L. var. napus) by HPLC-PDA-ESI(-)-MSn /HRMS. J. Agric. Food Chem. 2014, 62, 2935-2945. [CrossRef] [PubMed]

31. Kaur, C.; Kumar, K.; Anil, D.; Kapoor, H.C. Variations in antioxidant activity in broccoli (Brassica oleracea L.) cultivars. J. Food Biochem. 2007, 31, 621-638. [CrossRef]

32. Jaiswal, A.K.; Rajauria, G.; Abu-Ghannam, N.; Gupta, S. Phenolic composition, antioxidant capacity and antibacterial activity of selected Irish Brassica vegetables. Nat. Prod. Commun. 2011, 6, 1299-1304. [PubMed]

33. Dubie, J.; Stancik, A.; Morra, M.; Nindo, C. Antioxidant extraction from mustard (Brassica juncea) seed meal using high-intensity ultrasound. J. Food Sci. 2013, 78, 542-548. [CrossRef] [PubMed]

34. Siger, A.; Czubinski, J.; Kachlicki, P.; Dwiecki, K.; Lampart-Szczapa, E.; Nogala-Kalucka, M. Antioxidant activity and phenolic content in three lupin species. J. Food Compos. Anal. 2012, 25, 190-197. [CrossRef]

35. Kim, D.O.; Padilla-Zakour, O.I.; Griffiths, P.D. Flavonoids and antioxidant capacity of various cabbage genotypes at juvenile stage. J. Food Sci. 2004, 69, 9-13. [CrossRef]

36. The, S.S.; Bekhit, A.E.; Birch, J. Antioxidative polyphenols from defatted oilseed cakes: Effect of solvents. Antioxidants 2014, 3, 67-80. 
37. He, Q.; Zhang, Z.; Zhang, L. Anthocyanin accumulation, antioxidant ability and stability, and a transcriptional analysis of anthocyanin biosynthesis in purple heading Chinese cabbage (Brassica rapa L. ssp. pekinensis). J. Agric. Food Chem. 2016, 64, 132-145. [CrossRef] [PubMed]

38. Faudale, M.; Viladomat, F.; Bastida, J.; Poli, F.; Codina, C. Antioxidant activity and phenolic composition of wild, edible, and medicinal fennel from different Mediterranean countries. J. Agric. Food Chem. 2008, 56, 1912-1920. [CrossRef] [PubMed]

39. Brand-Williams, W.; Cuvelier, M.E.; Berset, C. Use of a free radical method to evaluate antioxidant activity. LWT Food Sci. Technol. 1995, 28, 25-30. [CrossRef]

40. Benzie, I.F.F.; Strain, J.J. The ferric reducing ability of plasma (FRAP) as a measure of "antioxidant power": The FRAP assay. Anal. Biochem. 1996, 239, 70-76. [CrossRef] [PubMed]

41. Re, R.; Pellegrini, N.; Proteggente, A.; Pannala, A.; Yang, M.; Rice-Evans, C. Antioxidant activity applying an improved ABTS radical cation decolorization assay. Free Radic. Biol. Med. 1999, 26, 1231-1237. [CrossRef]

42. Qu, C.; Zhao, H.; Fu, F.; Wang, Z.; Zhang, K.; Zhou, Y.; Wang, X.; Wang, R.; Xu, X.; Tang, Z.; et al. Genome-wide survey of flavonoid biosynthesis genes and gene expression analysis between black- and yellow-seeded Brassica napus. Front. Plant Sci. 2016, 7, 1755-1771. [CrossRef] [PubMed]

Sample Availability: Samples of the plant materials, for research only are available from the authors. 